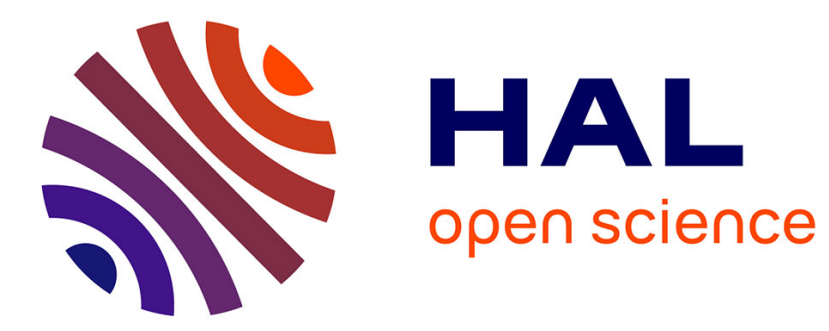

\title{
Extracellular vesicles as a platform to study cell-surface membrane proteins
}

Vincent Delauzun, Beatrice Amigues, Anais Gaubert, Philippe Leone, Magali Grange, Laurent Gauthier, Alain Roussel

\section{- To cite this version:}

Vincent Delauzun, Beatrice Amigues, Anais Gaubert, Philippe Leone, Magali Grange, et al.. Extracellular vesicles as a platform to study cell-surface membrane proteins. Methods, 2020, 180, pp.35-44. 10.1016/j.ymeth.2020.03.004 . hal-02989372

\section{HAL Id: hal-02989372 https://hal.science/hal-02989372}

Submitted on 10 Nov 2020

HAL is a multi-disciplinary open access archive for the deposit and dissemination of scientific research documents, whether they are published or not. The documents may come from teaching and research institutions in France or abroad, or from public or private research centers.
L'archive ouverte pluridisciplinaire HAL, est destinée au dépôt et à la diffusion de documents scientifiques de niveau recherche, publiés ou non, émanant des établissements d'enseignement et de recherche français ou étrangers, des laboratoires publics ou privés. 


\section{Extracellular vesicles as a new platform to study cell-surface membrane proteins}

Vincent Delauzun ${ }^{1,2}$, Beatrice Amigues ${ }^{1,2}$, Silvia Spinelli ${ }^{1,2}$, Anais Gaubert ${ }^{1,2}$, Philippe Leone ${ }^{1,2}$, Magali Grange ${ }^{1,2}$, Laurent Gauthier ${ }^{3}$, Alain Roussel ${ }^{1,2}$

${ }^{1}$ Architecture et Fonction des Macromolécules Biologiques, Aix-Marseille Université, UMR 7257, 163 Avenue de Luminy, Case 932, 13009 Marseille, France.

${ }^{2}$ Architecture et Fonction des Macromolécules Biologiques, Centre National de la Recherche Scientifique, UMR 7257, 163 Avenue de Luminy, Case 932, 13009 Marseille, France.

${ }^{3}$ Innate Pharma, 117 Avenue de Luminy, 13009 Marseille, France.

\section{Keywords}

Extracellular vesicle production

Membrane protein expression

Membrane protein-ligand interaction

Cell-surface receptor

Electron microscopy

Immunization

\section{Highlights}

Membrane protein interaction studies using extracellular vesicles

Extracellular vesicles as a tool for structure determination of membrane proteins

Extracellular vesicles use for immunization

\section{Abstract}

Producing intact recombinant membrane proteins for structural studies is an inherently challenging task due to their requirement for a cell-lipid environment. Most procedures developed involve isolating the protein by detergent solubilization and further reconstitutions into artificial membranes. These procedures are highly time consuming and suffer from further drawbacks, including low yields and high cost. 
We describe here an alternative method for rapidly obtaining recombinant cell-surface membrane proteins displayed on extracellular vesicles (EVs) derived from cells in culture. Interaction between these membrane proteins and ligands can be analyzed directly on EVs. Moreover, EVs can also be used for protein structure determination or immunization purposes.

\section{Introduction}

Cell-surface membrane proteins (MPs) [1] are involved in many biological processes such as, cell signalization [2] or viral infection [3], and play an essential role in regulation of the immune response [4]. These proteins which act as transporters, channels or receptors are preferred targets for drug researches and immunotherapies which are currently under development in pharmaceutical companies [5]. In this context, structure and interactions of these proteins with potential ligands such as therapeutic antibodies must be investigated. Entire cell-surface MPs can be produced and purified by extracting the protein out of the membrane through the use of detergents [6]. In addition to being long and expensive regarding to optimization needs, it is known that detergents do not stabilize membrane domains as efficiently as natural lipids in membranes, often leading to a partial to complete loss of activity/stability during protein extraction and purification [7]. Recent developments have made it possible to stabilize full-length MPs into artificial membranes such as nanodiscs or proteoliposomes [8] [9]. But these systems do not reflect the biochemical complexity of a real biological membrane and do not abrogate the use of detergents. Indeed, MPs embedded in a biological membrane such as a cellular lipid bilayer or a viral envelope can adopt complex orientations and conformations due to steric constrains depending on protein density and protein clustering pattern [10]. An alternative strategy may be used in case of monotopic MPs. Extracellular part without transmembrane domains can be expressed as a soluble protein which allows easier production and purification [11]. These truncated proteins can be used for structure determination by X-ray crystallography, singleparticle cryo-electron microscopy (EM) [12] or for immunization purposes [13]. One could notice that these soluble extracellular domains may display different biochemical characteristics compared to the original full-length molecules, making them a questionable study model [14]. 
All living cells release extracellular vesicles (EVs) in their environment. In animal species EVs are found in plasma, urine, milk, sweat, saliva or tears [15]. EVs can be classified into three sub-families: apoptotic bodies, exosomes and microvesicles according to their biogenesis [16]. Apoptotic bodies are produced during the apoptotic process, when cytoskeleton breaks up and causes the membrane to bulge outward [17]. Exosomes are generated through the endosomal pathway and accumulate inside multivesicular bodies before being release by exocytosis [18]. Microvesicles are formed by modification of the actin's network that drives budding the plasma membrane. This process is comparable to the mechanism involve for enveloped viruses exit of the cells [19]. MPs found on microvesicles surface are sorted in the cytoplasm by the ESCRT machinery [20]. Microvesicles size is about few tens to few hundred nanometers and they enclose soluble proteins, DNA and miRNA [21]. It has been recently shown that microvesicles can be used to display recombinant MPs on their surface for structure determination by cryo-electron tomography [22].

Here we describe a simple method to produce EVs covered by recombinant MPs of interest and their use for cell-surface MPs-ligands interaction analyses. Other EVs-based downstream applications are also discussed as new tools for cell-surface MPs studies.

\section{Material and methods}

\subsection{Plasmid construction}

\subsubsection{Vector}

pCAGGS-mcherry vector (Addgene plasmid \#41583) [23] was used to produce EVs. This vector was digested with EcoRI-HF (NEB \#R3101S) and Bglll (NEB \#R0144S) restriction enzymes in order to remove the mcherry gene out of the vector. Linearized vector was then separated by agarose gel $(1 \% \mathrm{~m} / \mathrm{v})$ electrophoresis and the DNA extracted band was purified by using a spin column from a DNA gel extraction kit (Biobasic \#EZ-10) according to manufacturer instructions.

\subsubsection{Genes}

Several genes coding for different cell-surface MPs have been cloned into the pCAGGS expression vector for this study. Zaire Ebola virus glycoprotein $\Delta 934-1389$ cDNA (Zebov GP) 
was ordered from Genscript company as a synthetic gene cloned into the pcDNA3.1 vector (Thermo Fisher \#V79020). This cDNA codes for the envelope's glycoprotein without its mucin domain [24]. Human Herpes Simplex Virus 1 glycoprotein B cDNA (HSV1 gB) [25] was synthetized by IDT Technologies company as a codon optimized (for mammalian expression system) gblock gene fragment flanked with $5^{\prime}$ and 3' hybridation sequences. Homo sapiens 5' ecto-nucleotidase cDNA (CD73) was kindly gifted as an amplified PCR product ready to be cloned by our collaborators from InnatePharma company. This cDNA encodes for the cellsurface CD73 human enzyme [26].

\subsubsection{Cloning}

Zebov GP and CD73 cDNAs were amplified by PCR with the Q-5 High Fidelity DNA polymerase (NEB \#M0491L) according to manufacturer instructions and PCR products were purified by agarose gel $(1 \% \mathrm{~m} / \mathrm{v})$ electrophoresis and the DNA extracted bands were purified using spin columns from a DNA gel extraction kit (Biobasic \#EZ-10).

PCR products (Zebov GP and CD73) and gblock gene fragment (HSV1 gB) were introduced into the linearized pCAGGS vector by using the In-Fusion HD cloning kit (Takara \#639649). Plasmids were then amplified under ampicillin selection after transformation of $\mathrm{E}$. coli RapidTrans TAM1 competent cells (Active Motif strain \#11096).

\section{Primers and hybridation sequences}

\begin{tabular}{|c|c|}
\hline Genes & \multicolumn{1}{|c|}{ Primers } \\
\hline Zebov GP & 5'-CATTTTGGCAAAGAATTGCCACCATGGGTGTTACAGGAATATTG-3' \\
Zebov GP & 5'-AAAAAGATCGAGATCTCAAAAGACAAATTTGCATATAC-3' \\
\hline CD73 & 5'-CATTTTGGCAAAGAATTGCCACCATGTGTCCCCGAGCCGCG-3' \\
CD73 & 5'-AAAAAGATCGAGATCTCATTGGTATAAAACAAAGATCACTGC-3' \\
\hline HSV1 gB & 5'-CATTTTGGCAAAGAATTGCCACC-3' \\
HSV1 gB & 5'-GATCTCGATCTTTTT-3' \\
\hline
\end{tabular}

\section{In-Fusion cloning parameters}




\begin{tabular}{|c|c|}
\hline Linearized pCAGGS vector & $100 \mathrm{ng}$ \\
\hline Insert & $50 \mathrm{ng}$ \\
\hline In-Fusion premix 5X & $2 \mu \mathrm{L}$ \\
\hline \multicolumn{2}{|c|}{ qsp H2O $10 \mu \mathrm{L}$} \\
\hline Incubate 15 min at $50^{\circ} \mathrm{C}$ \\
\hline
\end{tabular}

\subsection{EVs production}

\subsubsection{Cell culture}

HEK293 EBNA cells [27] were cultured in DMEM with high glucose $4.5 \mathrm{~g} / \mathrm{L}$ D-glucose, GlutaMAX'M and pyruvate (Gibco \#31966-021) supplemented with $10 \%$ (v/v) FBS (Sigma \#F9665). Cells were allowed to recover after thawing for at least one week, before performing recombinant EVs production. T175 flasks (Nunc Easyflask Thermoscientific \#159910) were used for expanding cells. Briefly cells were washed once with PBS without $\mathrm{CaCl} 2$ and $\mathrm{MgCl} 2$ (Gibco \#14190-136) and incubated with $0.25 \%$ Trypsin-EDTA solution (Gibco \#25200-056) for $3 \mathrm{~min}$ at $37^{\circ} \mathrm{C}$. Once one T175 flask reach $100 \%$ cell confluency it is enough to start EVs production.

\subsubsection{Transfection}

1) Detach the HEK293 EBNA cells from a $100 \%$ confluent T175 flask using $5 \mathrm{~mL}$ of 0.25 $\%$ trypsin-EDTA solution (Gibco \#25200-056).

2) Split the cells into 2 new T175 flasks with $35 \mathrm{~mL}$ of fresh complete DMEM medium.

3) Dilute $60 \mu \mathrm{g}$ of plasmid DNA in $5 \mathrm{~mL}$ of OptiMEM medium (Gibco \#31985-047) and $150 \mu \mathrm{L}$ of Lipofectamine 2000 (Invitrogen \#52887) in also $5 \mathrm{~mL}$ of OptiMEM medium. Mix solutions together and incubate $5 \mathrm{~min}$ at RT.

4) Dispatch $5 \mathrm{~mL}$ of transfection solution into each T175 and mix.

5) Incubate $5 \mathrm{~h}$ in a $\mathrm{CO} 2$ incubator at $37^{\circ} \mathrm{C}$ with $10 \% \mathrm{CO} 2$.

6) Replace serum-containing medium (DMEM) of each T175 flask with $25 \mathrm{~mL}$ of Freestyle293 serum-free medium with GlutaMAX'M (Gibco \#12338-018).

7) Incubate $96 \mathrm{~h}$ in a $\mathrm{CO} 2$ incubator at $37{ }^{\circ} \mathrm{C}$ with $10 \% \mathrm{CO} 2$ before collecting supernatant. 
Important considerations:

- HEK293 EBNA cells used instead HEK293 allows better EVs recovery

- Use of serum-free medium allows to eliminate bovine EVs

\subsection{EVs Purification}

\subsubsection{EVs enrichment}

Collect EVs containing supernatant $(50 \mathrm{~mL})$ and launch immediately differential centrifugation steps [28].

1) Centrifuge $10 \mathrm{~min}$ at $500 \mathrm{~g}$ at $4^{\circ} \mathrm{C}$ in order to eliminate residual cells. Keep supernatant.

2) Centrifuge $10 \mathrm{~min}$ at $3000 \mathrm{~g}$ at $4^{\circ} \mathrm{C}$ in order to eliminate bigger cellular contaminants. Keep supernatant.

3) Centrifuge $30 \mathrm{~min}$ at $10000 \mathrm{~g}$ at $4^{\circ} \mathrm{C}$. Keep supernatant.

4) Centrifuge $3 \mathrm{~h}$ at $110000 \mathrm{~g}$ at $4^{\circ} \mathrm{C}$ to pellet EVs in 2 ultracentrifuge tubes (Beckman coulter \#355616) with fixed angle rotor 50.2Ti on Optima LE-80k Ultracentrifuge from Beckman coulter (UC-1). Discard supernatant.

5) Wash EVs pellets carefully with $25 \mathrm{~mL}$ of PBS (Gibco \#14190-136) and centrifuge one more time $3 \mathrm{~h}$ at $110000 \mathrm{~g}$ at $4^{\circ} \mathrm{C}$ (UC-2). Discard supernatant.

6) Resuspend carefully each EVs pellet with $50 \mu \mathrm{L}$ of PBS (NaN3 $0.02 \%$ ) and store at $4^{\circ} \mathrm{C}$.

\subsubsection{EVs purification by SEC}

EVs were then purified by size exclusion chromatography using a HPLC system (Dionex Ultimate 3000 from Thermo Scientific). $100 \mu \mathrm{L}$ of EVs (absorbance at $280 \mathrm{~nm}$ between 2 to 3) were loaded on a Superdex 200 increase 10/300 GL prepacked gel filtration column (GE Healthcare Life Sciences \#28990944), which contains an agarose based-resin, eluted during $60 \mathrm{~min}$ with PBS (NaN3 $0.02 \%$ ) with a flow rate at $0.6 \mathrm{ml} / \mathrm{min}$ with UV detection at $280 \mathrm{~nm}$. EVs-containing SEC fractions $(100 \mu \mathrm{L})$ were collected and stored at $4^{\circ} \mathrm{C}$ for further analysis. For Cryo-EM experiments, SEC fractions were pooled and concentrated via ultrafiltration by passing SEC fractions through Vivaspin-500 10 kDa MWCO (GE Healthcare Life Sciences 
\#28932225) according to the manufacturer's instructions. The relative protein absorbance of each EV samples was measured at $280 \mathrm{~nm}$ by NanoDrop (Thermo Scientific).

Important considerations:

- Do not use any HPLC column pre-filter

- Do not use higher Vivaspin membrane cut-off [29]

\subsection{EVs Analysis}

\subsubsection{Dynamic Light Scattering}

$70 \mu \mathrm{L}$ of EVs (absorbance of fractions at $280 \mathrm{~nm}$ beetween 0.1 to 1) were analyzed by Dynamic Light Scattering (DLS) by using the Zetasizer Malvern Nano-S instrument. Hydrodynamic size distributions were measured in duplicate at $25^{\circ} \mathrm{C}$ during $40-80 \mathrm{sec}$ with a count rate between 200-600 kcps in a $3 \mathrm{~mm}$ light path quartz cuvette (Hellma \#105251005QS). The number of replicates conducted in one round was adapted automatically in the range of 10-20 measurements. The initial material Refractive Index (R.I.) was 1.45, while dispersant (PBS) properties were R.I. $=1.33$ and viscosity $=1.02 \mathrm{cP}$.

\subsubsection{Multi-Angle Light Scattering}

$50 \mu \mathrm{L}$ of SEC purified EVs (Abs280 = 0.3) were analyzed by SEC-MALS on a Superdex 200 increase 10/300 GL coupled with a multi-angle light scattering device (Dawn 8, Wyatt Technology) and an Optilab TrEX differential refractive index detector (Optilab TrEX, Wyatt Technology) operated with a HPLC system (Thermoscientific). Column was first equilibrated with PBS (NaN3 $0.02 \%$ ) for several hours, until the base line of the Optilab TrEX signal was stable. The light scattering and concentration data were analyzed in ASTRA to determine molecular weight and size distribution. Inter-detector band broadening and alignment were applied using the algorithms in ASTRA 7 (Wyatt Technology, USA) using BSA protein standard according to manufacturer-recommended methods. The $\mathrm{dn} / \mathrm{dc}$ used was $0.185 \mathrm{~mL} / \mathrm{g}$ since phosphate buffer was used for SEC. The temperature controlled-autosampler was kept at RT. 


\subsubsection{SDS-PAGE / Westernblot}

$50 \mu \mathrm{L}$ of SEC purified EVs (Abs280 = 1.4) were mix with DTT $(50 \mathrm{mM})$, and $1 X$ NuPage LDS sample buffer (Life Technologies \#NP0008). The samples were preheated at $80^{\circ} \mathrm{C}$ for 5 min and $20 \mu \mathrm{L}, 10 \mu \mathrm{L}$ or $5 \mu \mathrm{L}$ were loaded on a $4-20 \%$ Tris Glycine polyacrylamide gel (Nusep \#NG31-420). A molecular-weight size maker was used (Euromedex \#06U-0511). Electrophoresis was performed at $100 \mathrm{~V}$ (constant) and bands observed after staining with a $0.12 \%$ Coomassie blue solution (VWR, \#443286M). For western blot analysis, $20 \mu \mathrm{L}$ of EVs (Abs $=0.5)$ were loaded on SDS-PAGE gel and a pre-stained protein ladder was used (Euromedex \#06P-0111). After electrophoresis, the gel was blotted onto PVDF membranes (Immobilon-P 0.45 um \#IPVH00010, Merck Millipore). After blotting, membranes were blocked in PBS - 0.1 \% Tween-20 - 3\% BSA for 1 hour at room temperature. Membranes were then incubated with serum from a previously immunized llama (serum dilution 1/2000) for $1 \mathrm{~h}$ at room temperature. After two 10 minutes wash in PBS - $0.1 \%$ Tween-20 steps, membranes were incubated with a goat anti-Ilama-HRP conjugate antibody (dilution 1/5000) (Bethyl \#A160-100P) for $1 \mathrm{~h}$ at room temperature and the wash steps were repeated. Finally, membranes were subjected to HRP-detecting substrate (Immobilon Crescendo \#WBLUR0100, Merck Millipore) and imaged on Amersham Imager 680 System (GE Healthcare).

\subsubsection{Mass spectrometry}

Protein strips were cutted from the SDS-PAGE, digested with Trypsin and analyzed by MaldiTof/Tof (UltraFlexIII Bruker Daltonics). The peptides resulting from the digestion were compared to the list of theoretical enzymatic digestion peaks of the expected sequence. In parallel, a query of the Swissprot database by the Mascot software (Matrix Science) was carried out to detect contaminants.

\subsubsection{Biolayer Interferometry}

BLI experiments were performed using an Octet Red96 (FortéBio) with protein A-coated biosensor tips (Fortébio \#18-0028). A black bottom 96-well microplate (Greiner Bio-One \#655209) was filled with $200 \mu \mathrm{L}$ of solution (PBS buffer, antibodies or EVs) and agitated at 
$1000 \mathrm{rpm}$, and all experiments were temperature controlled at $25^{\circ} \mathrm{C}$. Tips were hydrated in PBS buffer for 1 hour at room temperature prior experiments. Baseline was performed with tips dipped into PBS buffer in the absence of EVs. Experiments were controlled with the software Data Acquisition 8.2 (FortéBio).

A mouse anti-CD73 antibody (IPH53, $5 \mu \mathrm{g} / \mathrm{mL}$ ) or a non-relevant antibody $(5 \mu \mathrm{g} / \mathrm{mL}$ ) were loaded on protein A sensors for $1 \mathrm{~min}$. The sensors were then rinsed in PBS buffer for 2.5 min to remove excess of antibody and then incubated with $200 \mu \mathrm{L}$ (Abs280 $=0.5$ ) of purified EVs expressing CD73 membrane protein for $4 \mathrm{~min}$ for the association step. The sensors were then dipped in PBS buffer for 8 min for the dissociation step. Measurements were analyzed with FortéBio Data analysis software version 7.1.

\subsubsection{Negative staining EM}

$6 \mu \mathrm{L}$ of SEC purified EVs (Abs280 = 0.2) were deposited onto a glow-discharged carboncoated grid (Ted Pella Formwar/Carbon, 300 mesh, Cu \#01813-F) using a PELCO easiGlow Glow Discharge Cleaning System (Ted Pella Inc., USA). Current: $25 \mathrm{~mA}$, time: $25 \mathrm{sec}$ and incubated for one minute. Sample excess was blotted off with a filter paper, rinsed with two $20 \mu \mathrm{L}$ drops of water and stained with $10 \mu \mathrm{L}$ of $2 \%$ uranyl acetate for $30 \mathrm{sec}$. Micrographs were recorded on a Veleta 2K x 2K CCD camera using a Tecnai T12 Spirit electron microscope (FEl Company) operated at $120 \mathrm{kV}$ and a magnification of $110000 \mathrm{X}$ or $220000 \mathrm{X}$.

\subsubsection{Cryo-EM}

$4 \mu \mathrm{l}$ of SEC purified EVs (Abs280 = 2) were deposited onto a glow-discharged perforated carbon-coated grid (Ted Pella LACEY Formvar/Carbon, 300 mesh, Cu \#01883-F) using a PELCO easiGlow Glow Discharge Cleaning System (Ted Pella Inc., USA). Current: 25 mA, time: $25 \mathrm{sec}$ and incubated for $5 \mathrm{sec}$. The excess liquid was backside blotted off with a filter paper (Whatman filter papers quality 5 \#1005-055) for $3 \mathrm{sec}$, the grid was then quickly plunged into liquid ethane using a Leica EM-GP cryo-chamber. EM grids were stored in cryo-boxes under liquid nitrogen until use, then mounted in a Gatan 626 cryo-holder and transferred in a Tecnai F20-FEG electron microscope (FEl Company) operated at $200 \mathrm{kV}$. Images were recorded with an USC1000-SSCCD Gatan camera and magnification of $110000 \mathrm{X}$. 


\subsection{Immunization}

\subsubsection{Llama immunization}

A llama (Lama glama) was immunized with purified HSV1 gB EVs (Ardèche-lamas France). 1 $\mathrm{mL}$ of HSV1 gB EVs (Abs280 = 0.1) were injected subcutaneously five times at 1-week intervals using incomplete Freund's adjuvant. Blood samples were collected aseptically 50 days after the last boost and serum isolated [30].

\subsubsection{Serum ELISA}

Pre-immune and immune sera were collected at day 0 and after 50 days of immunization. $100 \mu \mathrm{l}$ of SEC purified HSV1 gB-EVs (Abs280 = 0.1) were adsorbed on Nunc-Sorp plates (Thermo Fisher \#442404). The immune serum conversion was analyzed by ELISA, making serum dilution $1 / 250$ or $1 / 500$ in PBS and by using goat anti-llama-HRP conjugate (Bethyl \#A160-100P) and TMB reagent (Sigma \#T5525-50TAB) to develop the ELISA reaction. Absorbances of wells were recorded at $450 \mathrm{~nm}$ on a Safire 2 microplate reader (Tecan).

\section{Results and discussion}

We used several cell-surface MPs that are currently under studies in our laboratory to develop and validate our production process (Fig. 1). The Ebola and Herpes glycoproteins which are involved in the viral life cycle [31][32] are present on the surface of the viral envelope which derived from portions of the host-cell membranes. The human CD73 enzyme [33] which convert AMP to adenosine and which is over-expressed on tumor cells was also studied as part of our collaboration with Innate Pharma company [34].

\subsection{EVs production and purification}

Ebola glycoprotein was chosen as the first target to validate EVs production process due to its large size and easy-recognized shape by EM [35]. EVs purity was monitored during all the purification process by Dynamic Light Scattering (DLS) analysis. Hydrodynamic radius distribution by volume was preferred to distribution by intensity in order to report the relative percentages of high and low molecular weight species in solution [36] [37].

To produce EVs, mammalian cells were transfected with the pCAGGS vector containing the gene corresponding to the glycoprotein. Four days after transfection, EVs were first separated from dead cells and cellular debris and concentrated by differential 
centrifugations following the enrichment protocol described in section 2.3.1. DLS measurements after the two ultracentrigation steps (fig. 2 A) showed that most of soluble proteins and small protein aggregates with size under $40 \mathrm{~nm}$ that probably came from cell culture medium have been eliminated. EVs were further purified by size exclusion chromatography (Fig. 2 B). Collected fractions were grouped into three pools numbered from 1 to 3 and corresponding respectively to the beginning (between $6.5 \mathrm{~mL}$ and $7.5 \mathrm{~mL}$ ), the middle (between $7.5 \mathrm{~mL}$ and $8.5 \mathrm{~mL}$ ) and the ending part (between $8.5 \mathrm{~mL}$ and $9.5 \mathrm{~mL}$ ) of the EVs elution peak and were analyzed by DLS (Fig. 2 C). Large protein aggregates and cellular membrane contaminants with a size from $200 \mathrm{~nm}$ to $500 \mathrm{~nm}$ were found in pool-1 while smaller debris with a size under $40 \mathrm{~nm}$ were eluted at the end of the peak in pool-3. Proteins, DNA and RNA fragments that could remain after centrifugation steps [38] have been also separated and eluted later during SEC purification (between $14 \mathrm{~mL}$ and $20 \mathrm{~mL}$ ). DLS analyses of Pool-2 showed hydrodynamic radius distribution ranging from $25 \mathrm{~nm}$ to more than $100 \mathrm{~nm}$ with a Z-average of about $65 \mathrm{~nm}$. This pool was further analyzed by SECMALS, EM, SDS-PAGE and western blot.

\subsection{EVs characterization}

\subsubsection{Molecular mass, size and stability of EVs}

Purified EVs from pool-2 have been further characterized in terms of physical properties, stability and biochemical composition. Zebov GP-EVs concentration in pool-2 has been first evaluated by UV absorbance measurement at $280 \mathrm{~nm}$. We found a fairly good correlation rate between the absorbance values of different pool-2 samples and the number of EVs seen on negative staining EM grids. Therefore, we decided to take the UV absorbance measurement as a way to estimate the quantity of EVs in the samples. EVs from pool-2 were then analyzed by size-exclusion chromatography (SEC) coupled with multi-angle light scattering (MALS) detector and refractive index (RI) detector for the determination of molecular mass and size distribution (radius of gyration). SEC-MALS analysis of EVs (Fig. 3 A) revealed a molecular mass ranging from $80.10^{6}$ Da to $250.10^{6}$ Da with a value of around $150.10^{6} \mathrm{Da}$ at the middle of the peak. Radii of gyration of EVs were comprised between 50 $\mathrm{nm}$ and more than $100 \mathrm{~nm}$ with a value around $65 \mathrm{~nm}$ at the middle of the peak. These 
results were consistent with the size measurements performed by DLS during the previous purification steps. Interestingly, EVs proved to be highly stable at $4^{\circ} \mathrm{C}$ for months according to analysis by DLS and to observation by EM of stored samples. Indeed, DLS profiles of a sample taken during the purification step and after storage for 6 months at $4^{\circ} \mathrm{C}$ were clearly identical (Fig. 3 B). This result was important to validate the use of EVs as a membrane protein storage solution, which allows to always have samples ready for their use for downstream applications.

\subsubsection{Electron microscopy of EVs}

EM images of EVs from pool-2 have shown high level of EVs purity and validate our purification process. Size distribution of EVs previously investigated by DLS and SEC-MALS could also be confirmed by EM (Fig. 4 A). Typical cup-shaped of EVs were visualized by negative staining EM as expected. Furthermore, $90 \%$ of the EVs present on the EM grid were decorated with a MP. We were able to observe that the protein displayed all around the surface of the Zebov GP-EVs had the characteristic shape and size of the $10 \mathrm{~nm}$ height Ebola glycoprotein spike [39]. We were also able to observe EVs in their native state directly frozen in solution by cryo-electron microscopy (Fig. 4 B). EVs appeared as expected with shape of perfect spheres and decorated with a protein that were displayed and regularly anchored on the EV membrane. The number of over-expressed Zebov GP trimers per EV (radius size around $50 \mathrm{~nm}$ ) was estimated between 150 to 200.

\subsubsection{Zebov GP identification}

The presence of Zebov GP in EVs from pool-2 was then investigated by SDS-PAGE (Fig 5 A). Zebov GP is synthetized as single-chain precursor and transferred into the lumen of the endoplasmic reticulum where it forms trimers [39]. Then it is cleaved by furin enzyme into the receptor-binding GP1 and the fusion-mediating GP2 subunits and are incorporated into virions membrane to initiate infection. GP1 (which contains a mucin-like domain) and GP2 remained linked together by an inter chain disulfide bridge [40]. GP1 possesses a highly glycosylated mucin-like domain important for promoting viral infection. This domain has been removed in the present Zebov GP construct. GP1 and GP2 were identified by SDS-PAGE under reducing conditions. GP1 (around $60 \mathrm{kDa}$ ) and GP2 (around $25 \mathrm{kDa}$ ) appeared as 
characteristic smeared bands due to their high glycosylation states [41]. According to the SDS-PAGE gel, it appeared that Zebov GP represents a large part of the total mass of proteins contained in EVs. The presence of GP in EVs was then confirmed by western blot. We are engaged in a project that aims to generate neutralizing camelids antibodies (also named nanobodies) against Ebola virus. Our laboratory hosts a platform dedicated to the generation and selection of nanobodies against various antigens. As part of this service, a llama had already been immunized with the extracellular domain of the Zebov GP. The serum from this llama was used to confirm the presence of GP1 and GP2 in EVs by western blotting (Fig 5 B). Finally, an identification by peptide mass fingerprinting of the SDS-PAGE GP1 band was performed. The proteolytically generated peptides were investigated by determination of molecular masses. For protein identification, the experimentally obtained masses were compared with the theoretical peptide masses of proteins stored in databases by means of mass search programs [42]. In the total mass spectrum, $70 \%$ of peptides were identified with a sequence coverage of GP1 at $40 \%$. This partial coverage could be explained by the presence of glycosylation sites in the uncovered sequences, increasing the experimentally obtained masses of these glycosylated peptides. We could therefore conclude with certainty that Zebov GP was well expressed on EVs. Thanks to all these results, the same purification process was performed to produce CD73-EVs and HSV1 gBEVs.

\subsection{EVs applications}

It has been previously shown by $\operatorname{Dr}$ Zeev-Ben-Mordehai and colleagues from Oxford University, that EVs can be used for surface membrane protein structure determination by electron cryo-tomography [22]. Here we present two other applications that we investigated: the use of EVs for molecular interaction studies and their use for immunization for antibody generation.

\subsubsection{Interaction studies}

CD73 is over-expressed on tumor cells and leads to an abnormal increase in adenosine levels resulting in a down-regulation of the immune response. Innate Pharma company has recently developed an anti-CD73 antibody (IPH53) which blocks this enzyme an restore the immune system activity [34]. A human CD73 enzyme displayed on EVs (CD73-EVs) and IPH53 
antibody were used to investigate the ability of using EVs for ligand interaction studies. CD73-EVs were produced and purified as described above and firstly observed by EM (Fig. 6 A). CD73 proteins have been localized all around EVs with a size and shape ( $5 \mathrm{~nm}$ height by $13 \mathrm{~nm}$ width) consistent with protein structure predictions [43]. However, we noticed that a part of the purified EVs (around $30 \%$ ) did not display the CD73 protein unlike what we observed in the case of the Zebov GP protein for which more than $90 \%$ of the EVs were decorated. The total amount of CD73-EVs obtained was also 3 times less than the amount of Zebov GP-EVs obtained for a same production scale. This may due to lower transfection efficiency rate or to lower expression yield for this cell-surface MP construct. Thanks to molecular CD73-EV / antibody ratio optimizations we were able to fit conditions to get relevant EM images of the IPH53 binding on CD73-EVs. The EVs appeared clearly covered with an additional outer layer of IPH53 visible all around and on top of the EVs. (Fig 6 B). Many free antibodies were also visualized showing the excess of antibodies used.

We further investigated the interaction between IPH53 and CD73-EVs by biolayer interferometry experiment (Fig. 7). The association of CD73-EVs on protein A biosensor preloaded with IPH53 was monitored, which confirmed the interaction. We were unable to estimate any equilibrium dissociation constant (KD) between IPH53 antibody and CD73 antigen due to the corpuscular behavior of the EVs and their heterogeneous protein composition. Nevertheless, BLI experiments could be used to rapidly screen a large number of antibody interactions against cell-surface MPs displayed on EVs.

\subsubsection{Antibody generation}

Herpes virus entry into cells and membrane fusion require viral glycoproteins. Glycoprotein $B(g B)$ is one of the fusion proteins of HSV1 [44]. To investigate that EVs can be used for immunization purposes and antibody generation, HSV1 gB-EVs were produced and purified as describe previously and injected into a llama. Due to the high immunogenicity of EVs recently reported [45][46], we decided to injected to the llama 20-fold less gB protein (around $250 \mu \mathrm{g}$ ) than the amount of a soluble protein required for a standard immunization. Blood from llama was collected 50 days after the last injection and serum isolated. The immune response of the serum against the gB protein was evaluated by ELISA using HSV1 gB-EVs and compared to a pre-immune serum response. Absorbance values of wells were two times higher when serum from the immunized llama was used which confirmed 
antibody generation against the gB protein (Fig 8 A). The positive immune response of the serum against the gB protein (around $110 \mathrm{kDa}$ ) was also confirmed by the presence of the band at the right size on western blot revealed using the serum after immunization (Fig 8 B). Another band around $80 \mathrm{kDa}$ specific to HSV1 gB-EVs probably corresponding to a truncated form of gB protein was also observed.

\section{Conclusion}

We have developed a simple and fast protocol for the production of EVs coated with a cellsurface MP ready to use for downstream applications (Fig. 9). Due to their high stability, EVs give us a unique opportunity to store MPs in a biological environment. EVs can be used to investigate interaction between cell-surface MPs and potential ligands without the need for membrane protein extraction with detergent. Functional assays like enzymatic activity measurements can be performed directly on EVs. They can be also used as a powerful immunogenic antigen for antibody generation against MP extracellular part. Finally, due to their particular physico-chemical properties such as their size and composition, EVs constitute advantageous tools for structural studies by cryo-electron tomography of cellsurface MPs as close as possible to their natural state.

\section{Acknowledgements}

We thank Maria Mate from the AFMB laboratory (Marseille, France) for biophysical characterizations support and Patrick Fourquet from the Centre de Recherche en Cancérologie de Marseille for mass spectrometry analyses. We thank Marion Decossas from the Institut de chimie et biologie des membranes et des nano-objets (Bordeaux, France) for cryo-electron microscopy time allocation. The structural biology platform of the AFMB laboratory (Marseilles, France) was supported by the French Infrastructure for Integrated Structural Biology (FRISBI) ANR-10-INSB-05-01. This work was also supported by the Agence Nationale de la Recherche (ANR-14-EBOL-0001).

\section{References}

[1] K. R. Vinothkumar and R. Henderson, "Structures of membrane proteins," Q. Rev. Biophys., vol. 43, no. 1, pp. 65-158, 2010.

[2] H. E. Grecco, M. Schmick, and P. I. H. Bastiaens, "Signaling from the living plasma 
membrane," Cell, vol. 144, no. 6, pp. 897-909, 2011.

[3] M.-S. V. Laura de Armas-Rillo, "Membrane dynamics associated with viral infection," Rev. Med. Virol., vol. 26, no. January, pp. 146-160, 2016.

[4] E. M. Reuven, A. Fink, and Y. Shai, "Regulation of innate immune responses by transmembrane interactions: Lessons from the TLR family," Biochim. Biophys. ActaBiomembr., vol. 1838, no. 6, pp. 1586-1593, 2014.

[5] E. Narni-Mancinelli et al., "Complement factor P is a ligand for the natural killer cellactivating receptor NKp46," Sci. Immunol., vol. 2, no. 10, 2017.

[6] V. Kotov et al., "High-throughput stability screening for detergent-solubilized membrane proteins," Sci. Rep., vol. 9, no. 1, pp. 1-19, 2019.

[7] R. Matar-Merheb et al., "Structuring detergents for extracting and stabilizing functional membrane proteins," PLoS One, vol. 6, no. 3, pp. 1-10, 2011.

[8] M. Yokogawa, M. Fukuda, and M. Osawa, "Nanodiscs for structural biology in a membranous environment," Chem. Pharm. Bull., vol. 67, no. 4, pp. 321-326, 2019.

[9] L. Console, M. Tolomeo, M. Colella, M. Barile, and C. Indiveri, "Reconstitution in Proteoliposomes of the Recombinant Human Riboflavin Transporter 2 (SLC52A2) Overexpressed in E. coli," Int. J. Mol. Sci., vol. 20, no. 18, p. 4416, 2019.

[10] M. F. Garcia-Parajo, A. Cambi, J. A. Torreno-Pina, N. Thompson, and K. Jacobson, "Nanoclustering as a dominant feature of plasma membrane organization," J. Cell Sci., vol. 127, no. 23, pp. 4995-5005, 2014.

[11] M. Pavsic and B. Lenarcic, "Expression, crystallization and preliminary X-ray characterization of the human epithelial cell-adhesion molecule ectodomain," Acta Crystallogr. Sect. F Struct. Biol. Cryst. Commun., vol. 67, no. 11, pp. 1363-1366, 2011.

[12] E. V. Shtykova et al., "The dimeric ectodomain of the alkali-sensing insulin receptorrelated receptor (ectolRR) has a drop-like shape," J. Biol. Chem., p. jbc.RA119.010390, 2019.

[13] K. El Bakkouri et al., "Universal Vaccine Based on Ectodomain of Matrix Protein 2 of Influenza A: Fc Receptors and Alveolar Macrophages Mediate Protection," J. Immunol., vol. 186, no. 2, pp. 1022-1031, 2011.

[14] E. E. H. Tran et al., "Spatial Localization of the Ebola Virus Glycoprotein Mucin-Like Domain Determined by Cryo-Electron Tomography," J. Virol., vol. 88, no. 18, pp. 10958-10962, 2014. 
[15] C. V. Harding, J. E. Heuser, and P. D. Stahl, “Exosomes: Looking back three decades and into the future," J. Cell Biol., vol. 200, no. 4, pp. 367-371, 2013.

[16] E. van der Pol, A. N. Boing, P. Harrison, A. Sturk, and R. Nieuwland, "Classification, Functions, and Clinical Relevance of Extracellular Vesicles," Pharmacol. Rev., vol. 64, no. 3, pp. 676-705, 2012.

[17] S. Caruso and I. K. H. Poon, "Apoptotic cell-derived extracellular vesicles: More than just debris," Front. Immunol., vol. 9, no. JUN, 2018.

[18] A. Takahashi et al., "Exosomes maintain cellular homeostasis by excreting harmful DNA from cells," Nat. Commun., vol. 8, no. May, pp. 1-14, 2017.

[19] G. Van Niel, G. D'Angelo, and G. Raposo, "Shedding light on the cell biology of extracellular vesicles," Nat. Rev. Mol. Cell Biol., vol. 19, no. 4, pp. 213-228, 2018.

[20] L. Christ, C. Raiborg, E. M. Wenzel, C. Campsteijn, and H. Stenmark, "Cellular functions and molecular mechanisms of the ESCRT membrane-scission machinery," Trends Biochem. Sci., vol. 42, no. 1, pp. 42-56, 2017.

[21] A. Cvjetkovic et al., "Detailed Analysis of Protein Topology of Extracellular VesiclesEvidence of Unconventional Membrane Protein Orientation," Sci. Rep., vol. 6, no. November, pp. 1-12, 2016.

[22] T. Zeev-Ben-Mordehai, D. Vasishtan, C. A. Siebert, C. Whittle, and K. Grünewald, "Extracellular Vesicles: A Platform for the Structure Determination of Membrane Proteins by Cryo-EM," Structure, vol. 22, no. 11, pp. 1687-1692, 2014.

[23] H. Niwa, K. Yamamura, and J. Miyazaki, "Efficient selection for high-expression transfectants with a novel eukaryotic vector," Gene, vol. 108, pp. 193-199, 1991.

[24] O. Martinez, L. Tantral, N. Mulherkar, K. Chandran, and C. F. Basler, "Impact of Ebola mucin-like domain on antiglycoprotein antibody responses induced by Ebola virus-like particles," J. Infect. Dis., vol. 204, no. SUPPL. 3, pp. 825-832, 2011.

[25] T. Zeev-Ben-Mordehai et al., "Two distinct trimeric conformations of natively membrane-anchored full-length herpes simplex virus 1 glycoprotein B," Proc. Natl. Acad. Sci. U. S. A., vol. 113, no. 15, pp. 4176-4181, 2016.

[26] P. J. Schuler et al., "Human CD4+CD39+ regulatory T cells produce adenosine upon coexpression of surface CD73 or contact with CD73+ exosomes or CD73+ cells," Clin. Exp. Immunol., vol. 177, no. 2, pp. 531-543, 2014.

[27] R. Tom, L. Bisson, and Y. Durocher, "Culture of HEK293-EBNA1 cells for production of 
recombinant proteins," Cold Spring Harb. Protoc., vol. 3, no. 3, pp. 1-5, 2008.

[28] M. A. Livshts et al., "Isolation of exosomes by differential centrifugation: Theoretical analysis of a commonly used protocol," Sci. Rep., vol. 5, no. October, pp. 1-13, 2015.

[29] B. J. Benedikter et al., "Ultrafiltration combined with size exclusion chromatography efficiently isolates extracellular vesicles from cell culture media for compositional and functional studies," Sci. Rep., vol. 7, no. 1, pp. 1-13, 2017.

[30] A. Desmyter, S. Spinelli, A. Roussel, and C. Cambillau, "Camelid nanobodies: Killing two birds with one stone," Curr. Opin. Struct. Biol., vol. 32, pp. 1-8, 2015.

[31] C. L. Hunt, N. J. Lennemann, and W. Maury, "Filovirus entry: A novelty in the viral fusion world," Viruses, vol. 4, no. 2, pp. 258-275, 2012.

[32] F. C. Bender, J. C. Whitbeck, H. Lou, G. H. Cohen, and R. J. Eisenberg, "Herpes Simplex Virus Glycoprotein B Binds to Cell Surfaces Independently of Heparan Sulfate and Blocks Virus Entry," J. Virol., vol. 79, no. 18, pp. 11588-11597, 2005.

[33] M. Adzic and N. Nedeljkovic, "Unveiling the role of Ecto-5'-nucleotidase/CD73 in astrocyte migration by using pharmacological tools," Front. Pharmacol., vol. 9, no. MAR, pp. 1-19, 2018.

[34] I. Perrot et al., "Blocking Antibodies Targeting the CD39/CD73 Immunosuppressive Pathway Unleash Immune Responses in Combination Cancer Therapies," Cell Rep., vol. 27, no. 8, pp. 2411-2425.e9, 2019.

[35] J. E. Lee and E. Ollmann Saphire, "Ebolavirus glycoprotein structure and mechanism of entry," Future Virol., vol. 4, no. 6, pp. 621-635, 2009.

[36] J. Stetefeld, S. A. McKenna, and T. R. Patel, "Dynamic light scattering: a practical guide and applications in biomedical sciences," Biophys. Rev., vol. 8, no. 4, pp. 409-427, 2016.

[37] C. Washington, Particle size analysis in pharmaceutics and other industries : theory and practice. New York (N.Y.) : Ellis Horwood, 1992.

[38] A. Cvjetkovic, J. Lötvall, and C. Lässer, "The influence of rotor type and centrifugation time on the yield and purity of extracellular vesicles," J. Extracell. Vesicles, vol. 3, no. 1, pp. 1-11, 2014.

[39] D. R. Beniac and B. F. Timothy, "Structure of the Ebola virus glycoprotein spike within the virion envelope at 11 Å resolution," Sci. Rep., vol. 7, no. April, pp. 1-8, 2017.

[40] W. Weissenhorn, A. Carfí, K. H. Lee, J. J. Skehel, and D. C. Wiley, "Crystal structure of 
the Ebola virus membrane fusion subunit, GP2, from the envelope glycoprotein ectodomain," Mol. Cell, vol. 2, no. 5, pp. 605-616, 1998.

[41] B. Wang et al., "Mechanistic understanding of N-glycosylation in Ebola virus glycoprotein maturation and function," J. Biol. Chem., vol. 292, no. 14, pp. 5860-5870, 2017.

[42] B. Thiede et al., "Peptide mass fingerprinting," Methods, vol. 35, no. 3 SPEC.ISS., pp. 237-247, 2005.

[43] K. Knapp, M. Zebisch, J. Pippel, A. El-Tayeb, C. E. Müller, and N. Sträter, “Crystal structure of the human ecto-5'-nucleotidase (CD73): Insights into the regulation of purinergic signaling," Structure, vol. 20, no. 12, pp. 2161-2173, 2012.

[44] U. E. Maurer et al., "The structure of herpesvirus fusion glycoprotein B-bilayer complex reveals the protein-membrane and lateral protein-protein interaction," Structure, vol. 21, no. 8, pp. 1396-1405, 2013.

[45] C. J. E. Wahlund, G. Güclüler, S. Hiltbrunner, R. E. Veerman, T. I. Näslund, and S. Gabrielsson, "Exosomes from antigen-pulsed dendritic cells induce stronger antigenspecific immune responses than microvesicles in vivo," Sci. Rep., vol. 7, no. 1, pp. 1-9, 2017.

[46] P. B. Devhare and R. B. Ray, "A novel role of exosomes in the vaccination approach," Ann. Transl. Med., vol. 5, no. 1, pp. 23-23, 2017. 
Figure legends

Figure 1: EVs production workflow

Figure 2: EVs purification analyses

A) Hydrodynamic radius distribution of samples analyzed by dynamic light scattering (DLS) after each purification step: the first ultracentrifugation (UC-1, circle), the second ultracentrifugation (UC-2, square) and the size exclusion chromatography (SEC, triangle). B) SEC from the UC-2 sample: pool-1 (6.5 mL-7.5 mL, green box), pool-2 (7.5 mL-8.5 mL, black box), pool-3 (8.5 mL-9.5 mL, red box). C) Hydrodynamic radius distribution of each part of the SEC elution peak (pool-1: green line, pool-2: black line and pool-3: red line) analyzed by DLS.

Figure 3: SEC-MALS and DLS analyses of EVs.

A) Molecular mass (blue) and radius of gyration (orange) of Zebov GP-EVs from pool-2 analyzed by SEC-MALS. B) EVs size distribution monitored by DLS of a UC-2 sample (full) and after a six-months storage period at $4^{\circ} \mathrm{C}$ (UC-2 / 6 months, cross).

Figure 4: Electron microscopy (EM) of EVs.

A) Negative stain EM image (left panel) and close-up view (right panel) of purified Zebov GPEVs (magnification $110000 \mathrm{X}$ ). Zebov GP proteins (white arrows) are displayed all around EVs. B) Cryo-EM image (left panel) and close-up view (right panel) of purified Zebov GP-EVs (magnification $110000 \mathrm{X}$ ). Zebov GP proteins (black arrows) are displayed all around EVs.

Figure 5: Zebov GP identification.

A) $20 \mu \mathrm{L}, 10 \mu \mathrm{L}$ or $5 \mu \mathrm{L}$ of Zebov GP-EVs (Abs280 = 1.4) from pool-2 analyzed by SDS-PAGE and revealed by Coomassie blue staining. B) $20 \mu \mathrm{L}$ (Abs280 $=0.5$ ) of different EVs from pool2 (lane 1: HSV1 GPB-EVs, lane 2: CD73-EVs, lane 3: Zebov GP-EVs) were analyzed by western blot and revealed with a llama serum.

Figure 6: Electron microscopy (EM) of CD73-EVs with IPH53 antibody.

A) Negative stain EM image of purified CD73-EVs (left panel) and close-up view (right panel) of CD73 protein (white arrows). The upper vesicle is decorated with CD73 while the lower one is not (magnification $220000 \mathrm{X}$ ). B) Negative stain EM image of a purified CD73-EVs (left panel) covered with IPH53 antibody (black arrows) and close-up view (right panel). Free IPH53 antibody can be also observed (black boxes).

Figure 7: CD73-EVs and IPH53 interaction analysis by biolayer interferometry (BLI).

Biolayer interferometry analysis of IPH53 binding to CD73-EVs (black line) using protein A biosensors. A non-relevant antibody was used as negative control (red line). Experiments were divided into 4 different phases: the baseline acquisition, the loading of antibodies on the sensor, another baseline acquisition corresponding to a washing step of the sensors, the association and the dissociation phase.

Figure 8: Llama immunization with HSV1 gB-EVs 
A) Pre-immune serum (J0, black bar) and serum collected 50 days after HSV1 gB-EVs last injection (J50, white bar) at two different dilutions (1/250 and $1 / 500)$ were analyzed by ELISA using $100 \mu \mathrm{L}$ of purified HSV1 gB-EVs (Abs280 = 0.1). B) $20 \mu \mathrm{L}$ (Abs280 =0.5) of purified EVs (lane 1: HSV1 gB-EVs, lane 2: CD73-EVs) were analyzed by western blot and revealed with serum collected 50 days (J50) after HSV1 gB-EVs last injection.

Figure 9: Downstream applications summarize

EVs can be used for several downstream applications: biophysical studies (e.g. interaction studies with potential ligands) or functional studies (e.g. enzymatic activity studies), structure resolution (e.g. by cryo-electron tomography) or immunization purposes (e.g. llama immunization for nanobodies generation). 
Figure 1

Cell-surface MP cDNA synthesis and cloning

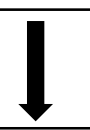

Cell culture and transfection

Supernatant recovery and EVs enrichment

EVs purification

I

EVs quality control by EM

I

Downstream applications 
Figure 2
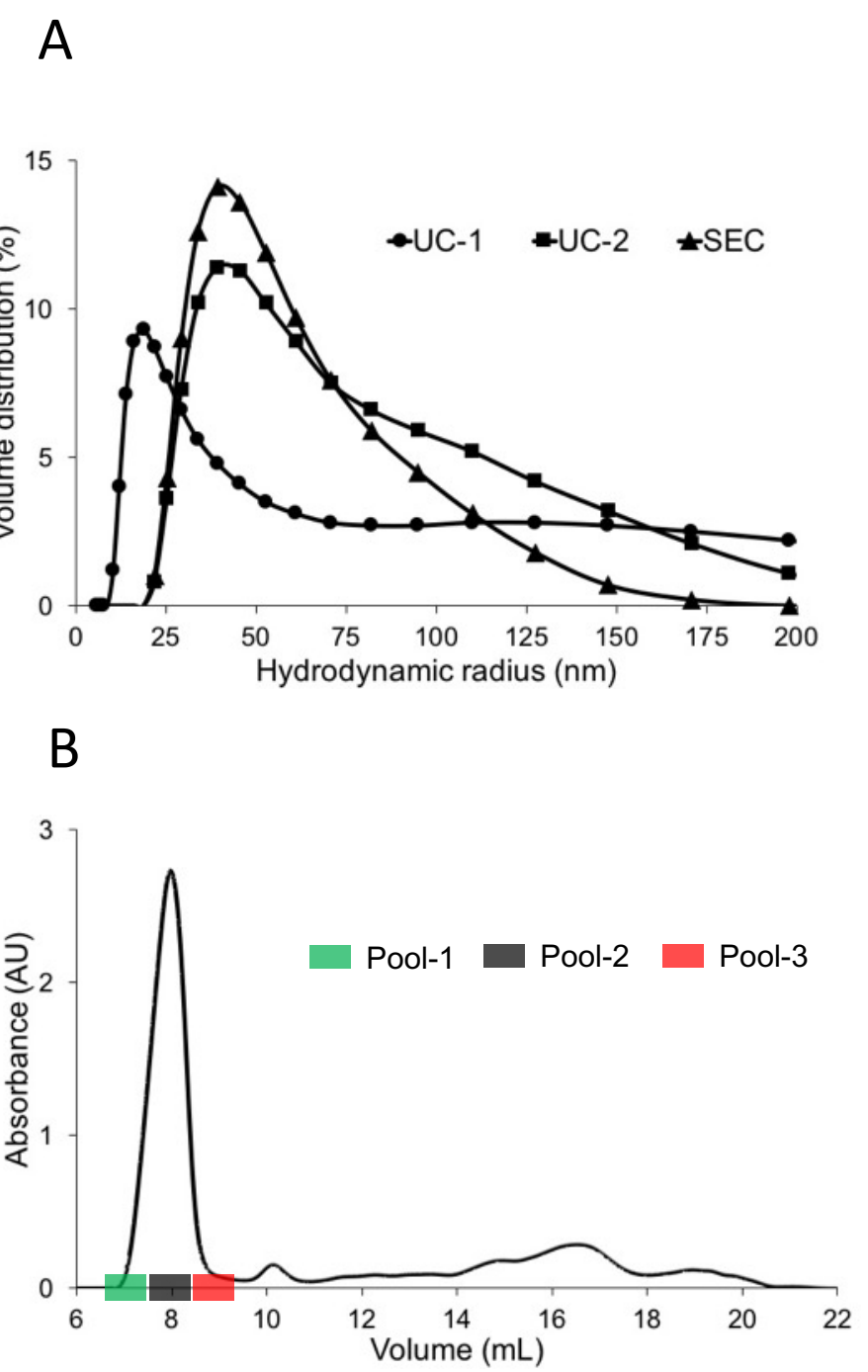

C

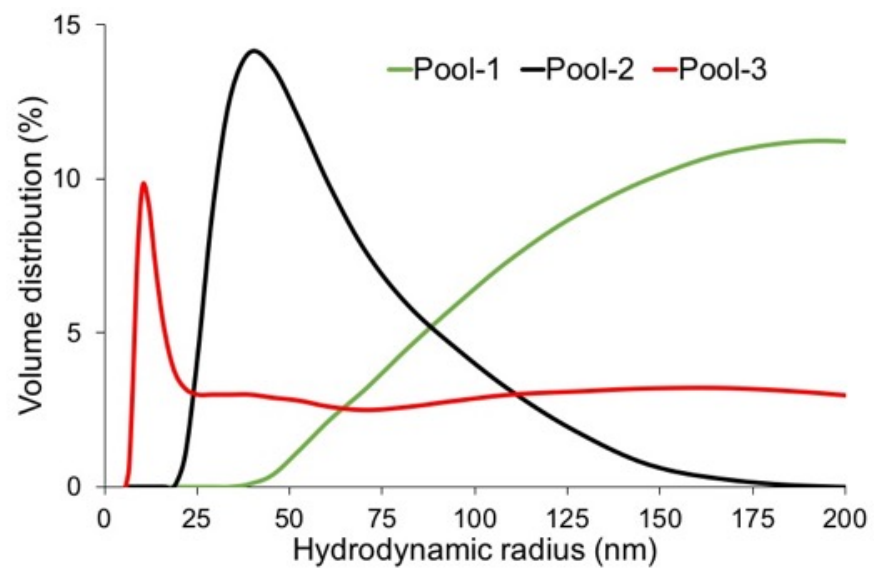


Figure 3

\section{A}

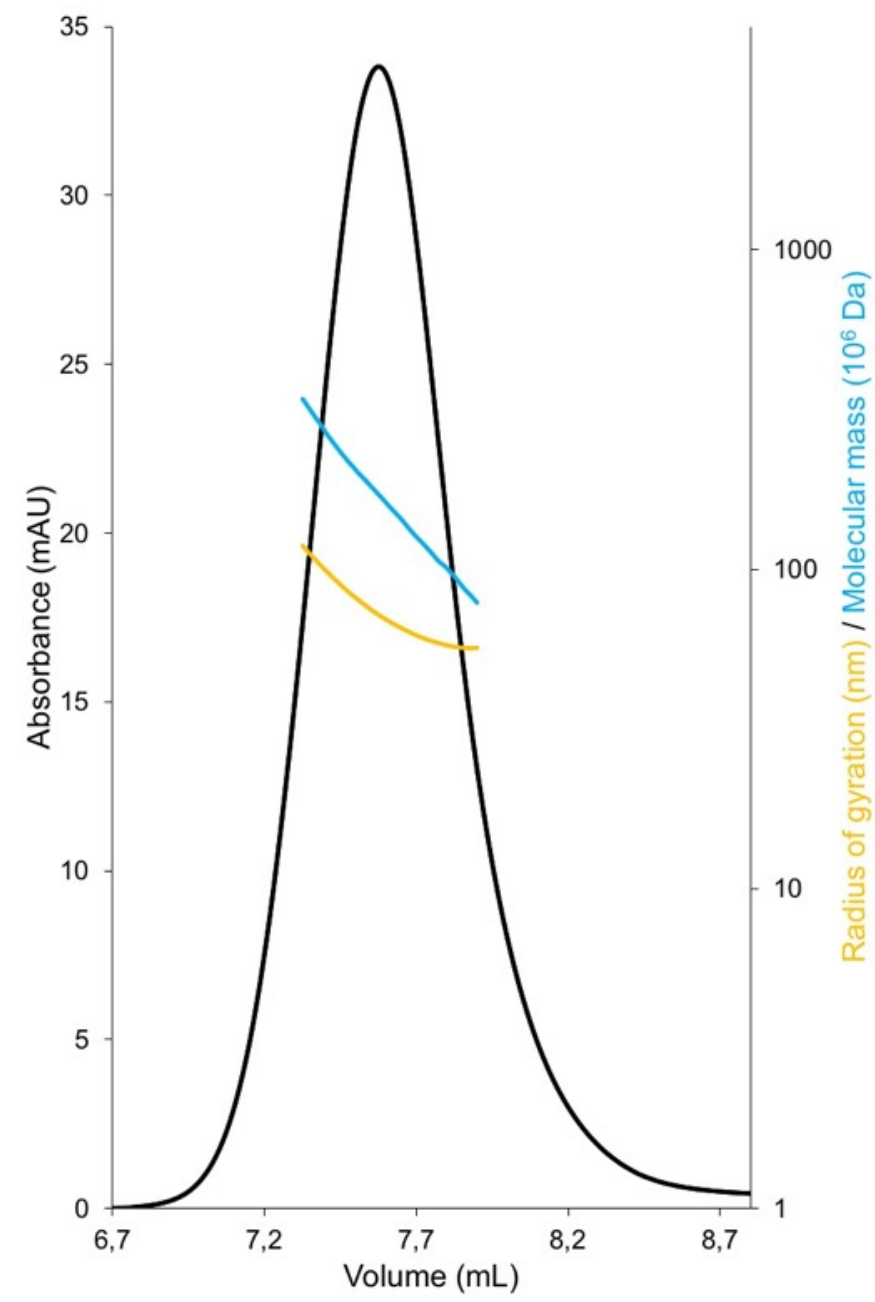

B

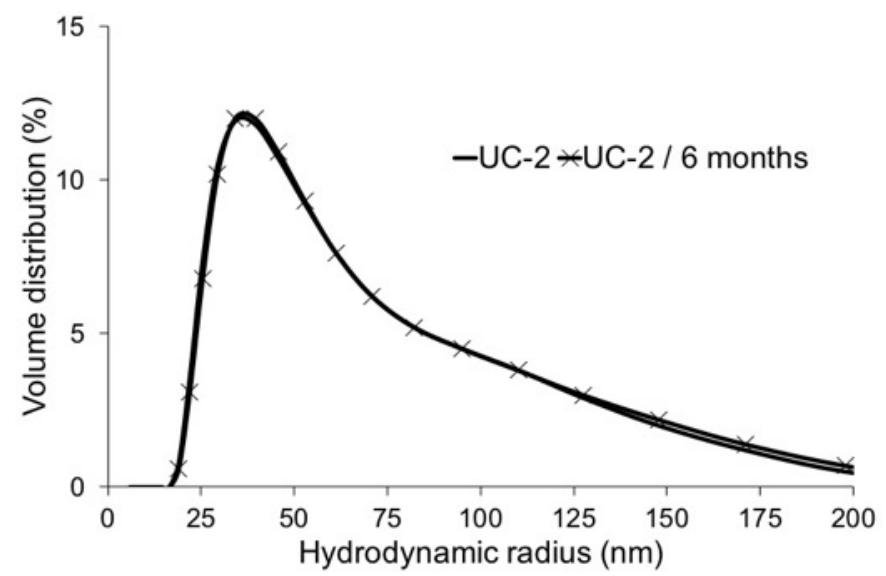




\section{Figure 4}

A
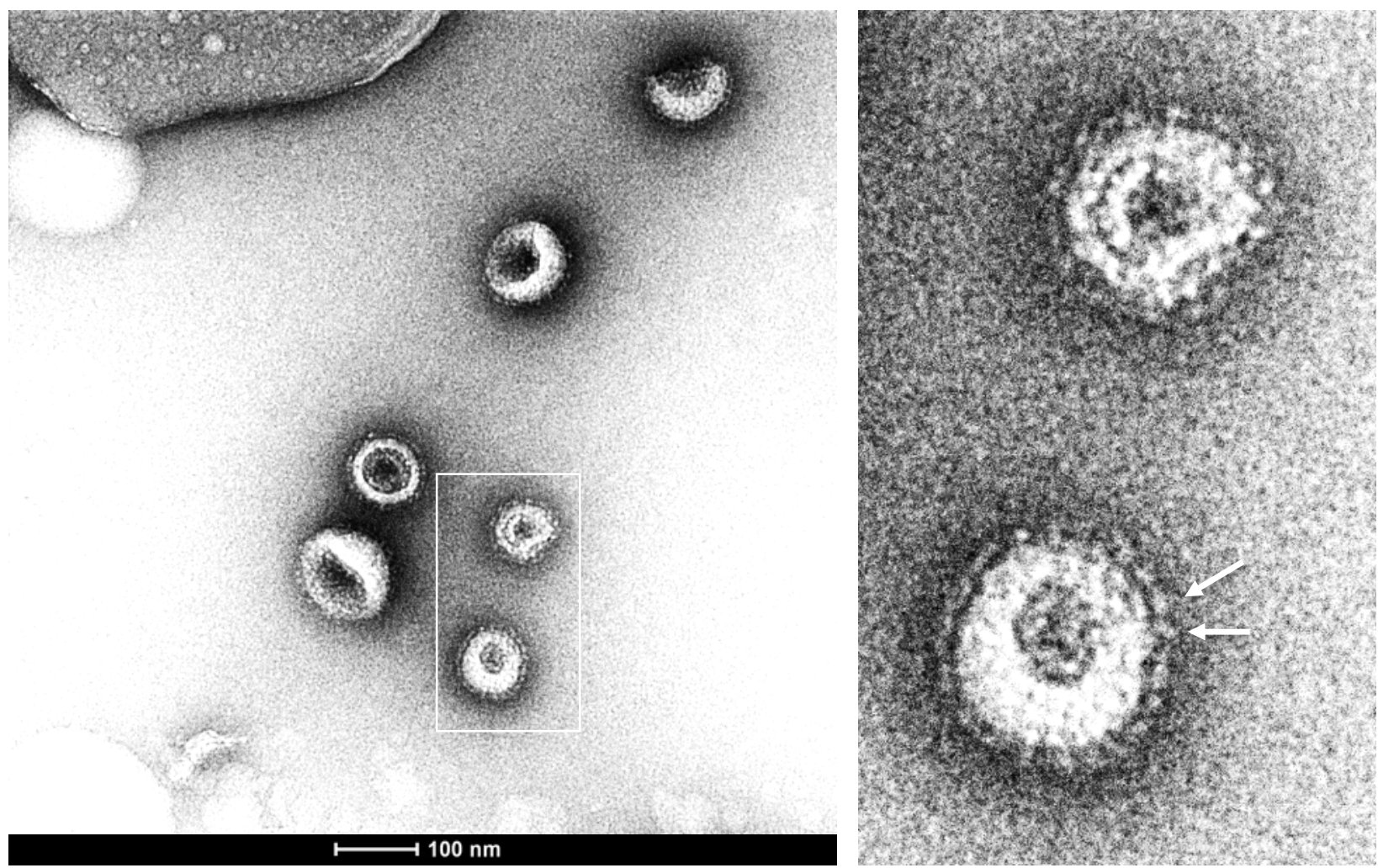

B
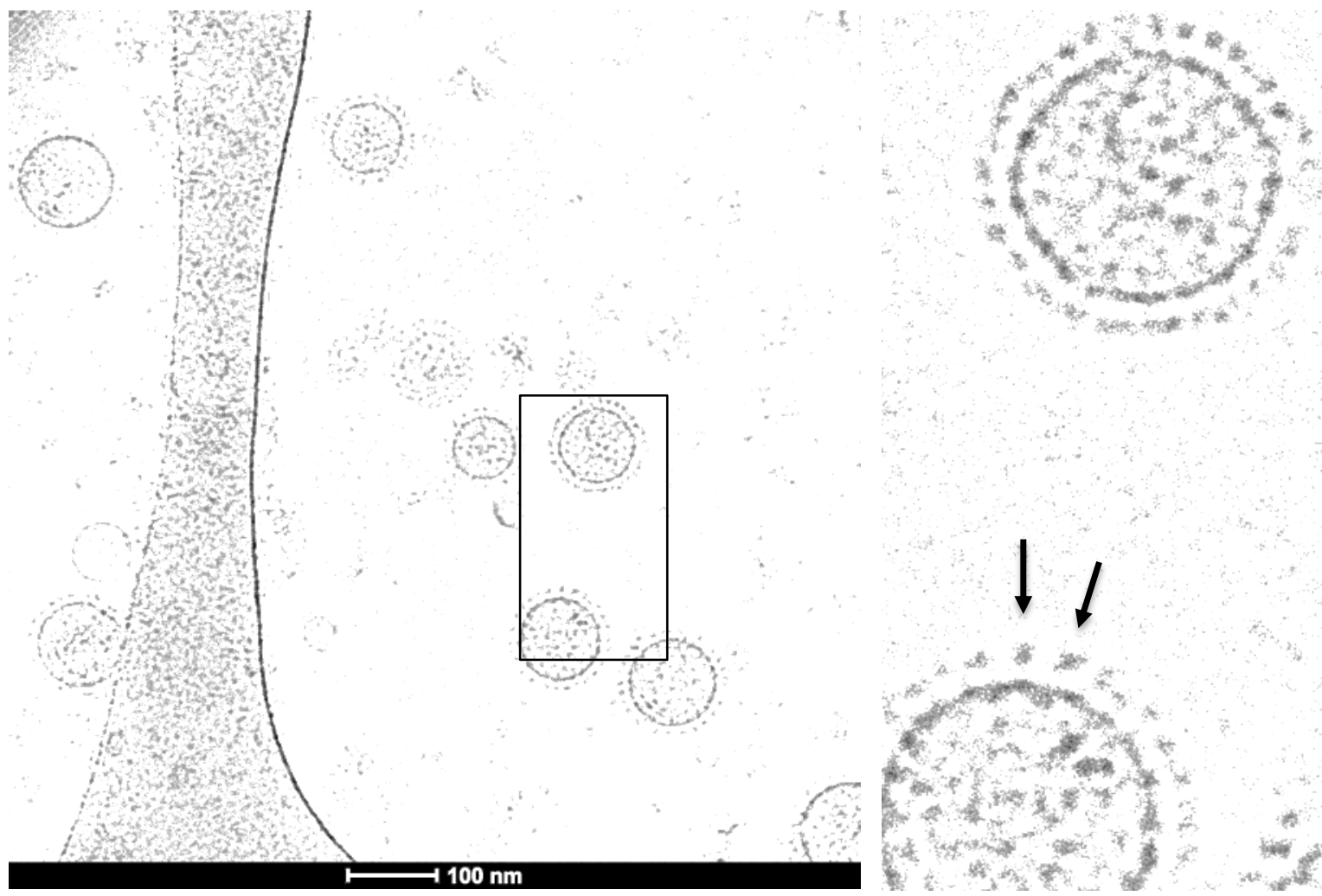

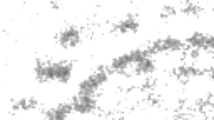




\section{Figure 5}

A

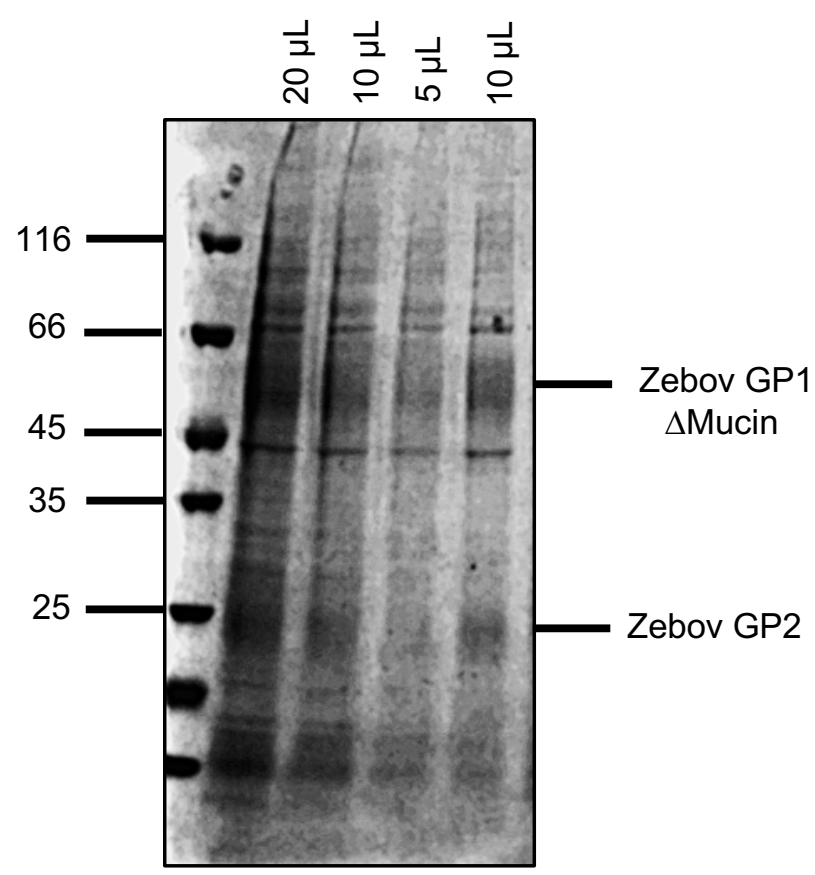

B

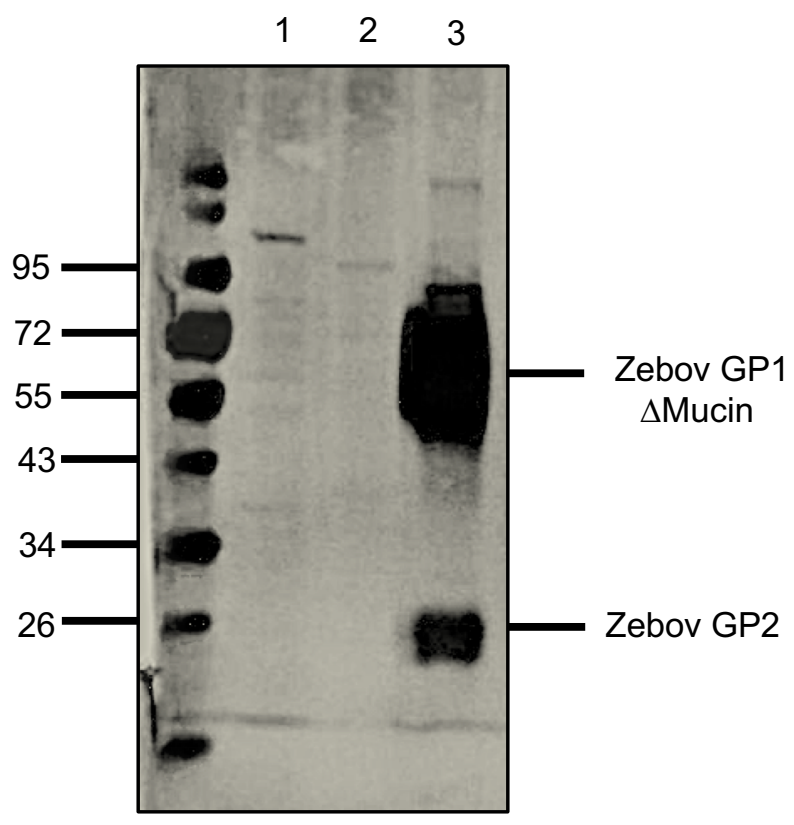




\section{Figure 6}

A

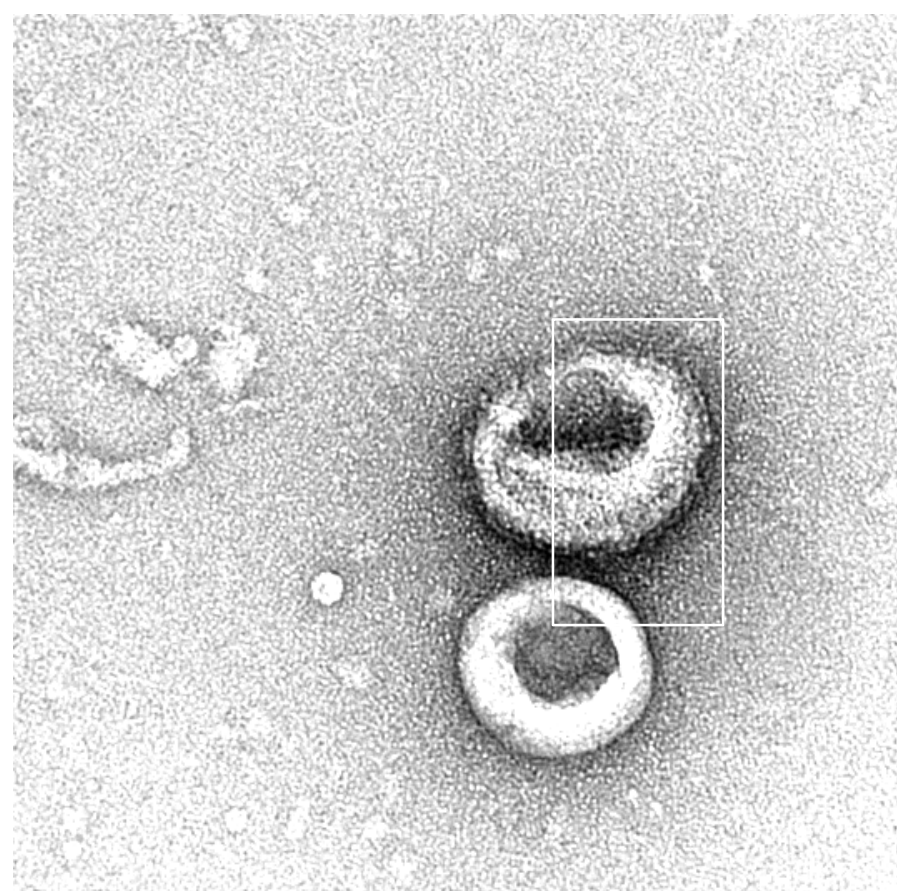

$-50 \mathrm{~nm}$

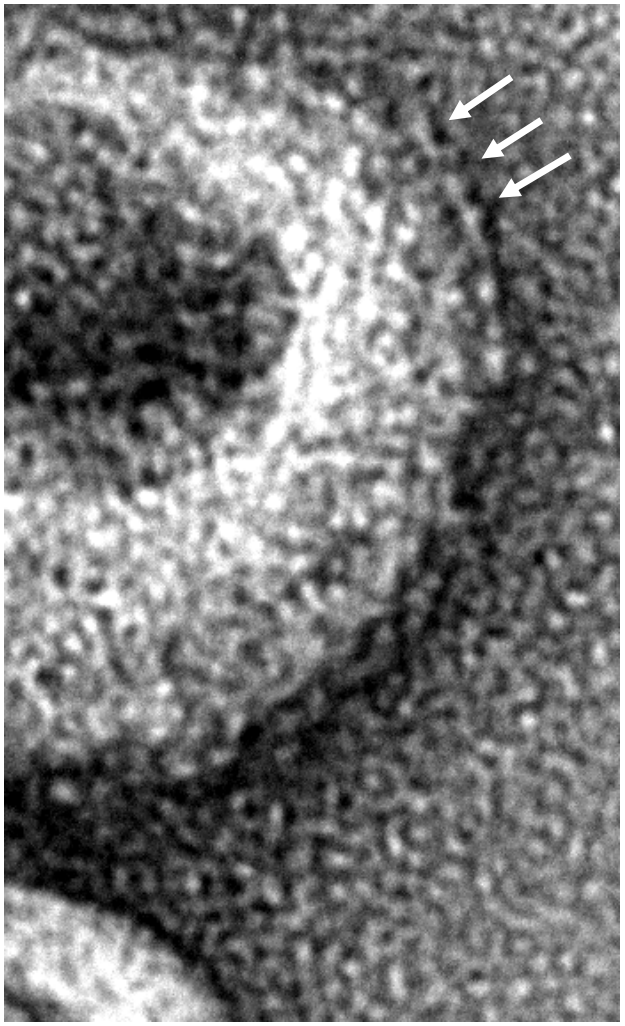

B
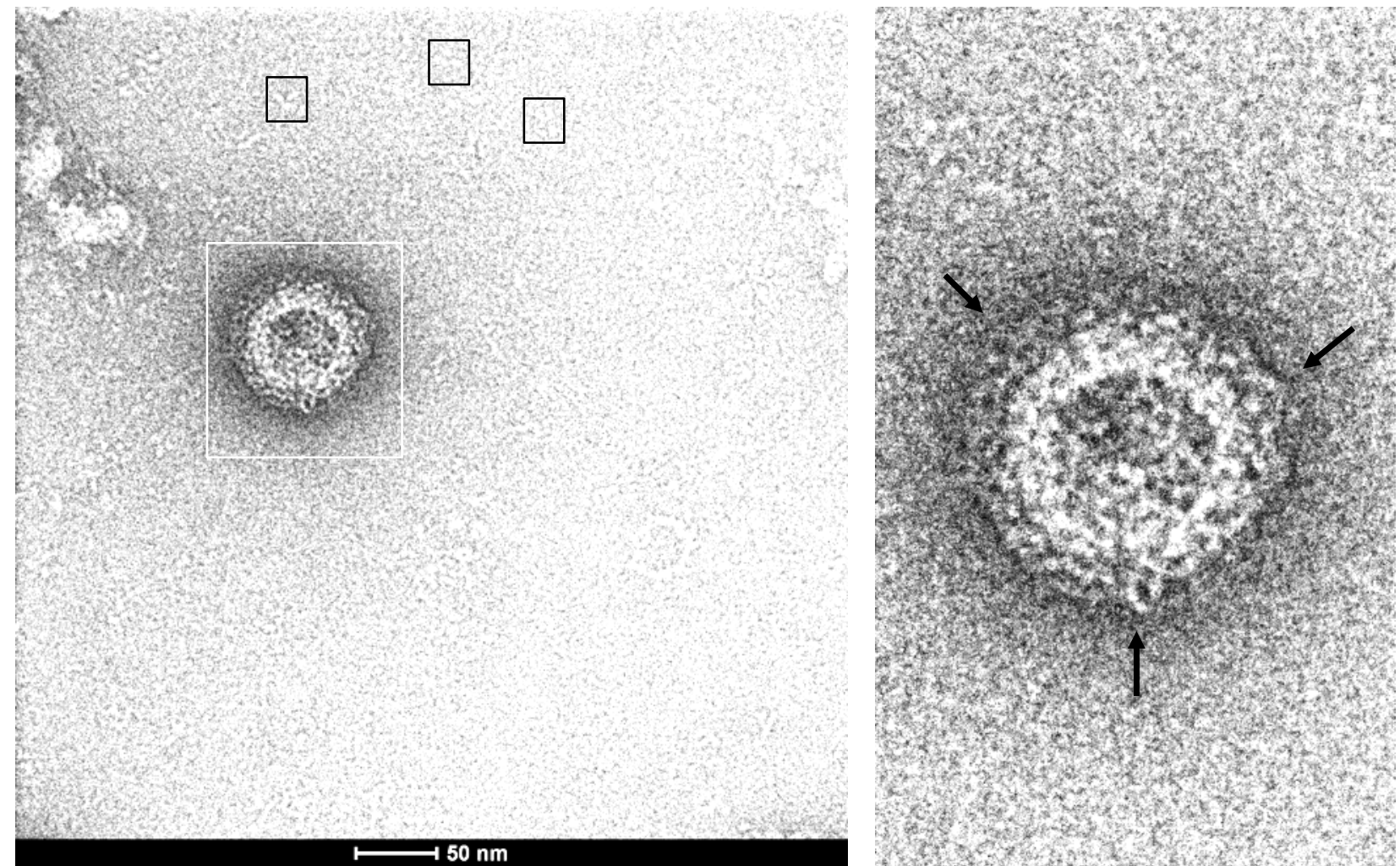
Figure 7

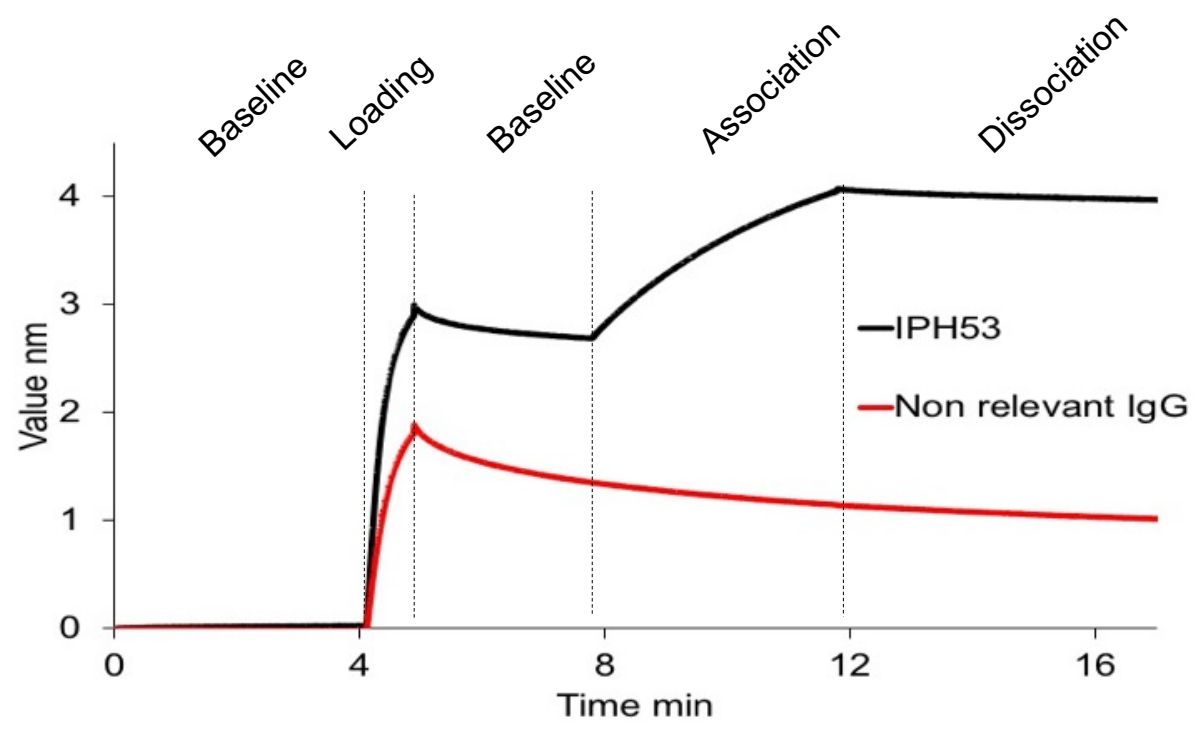


Figure 8
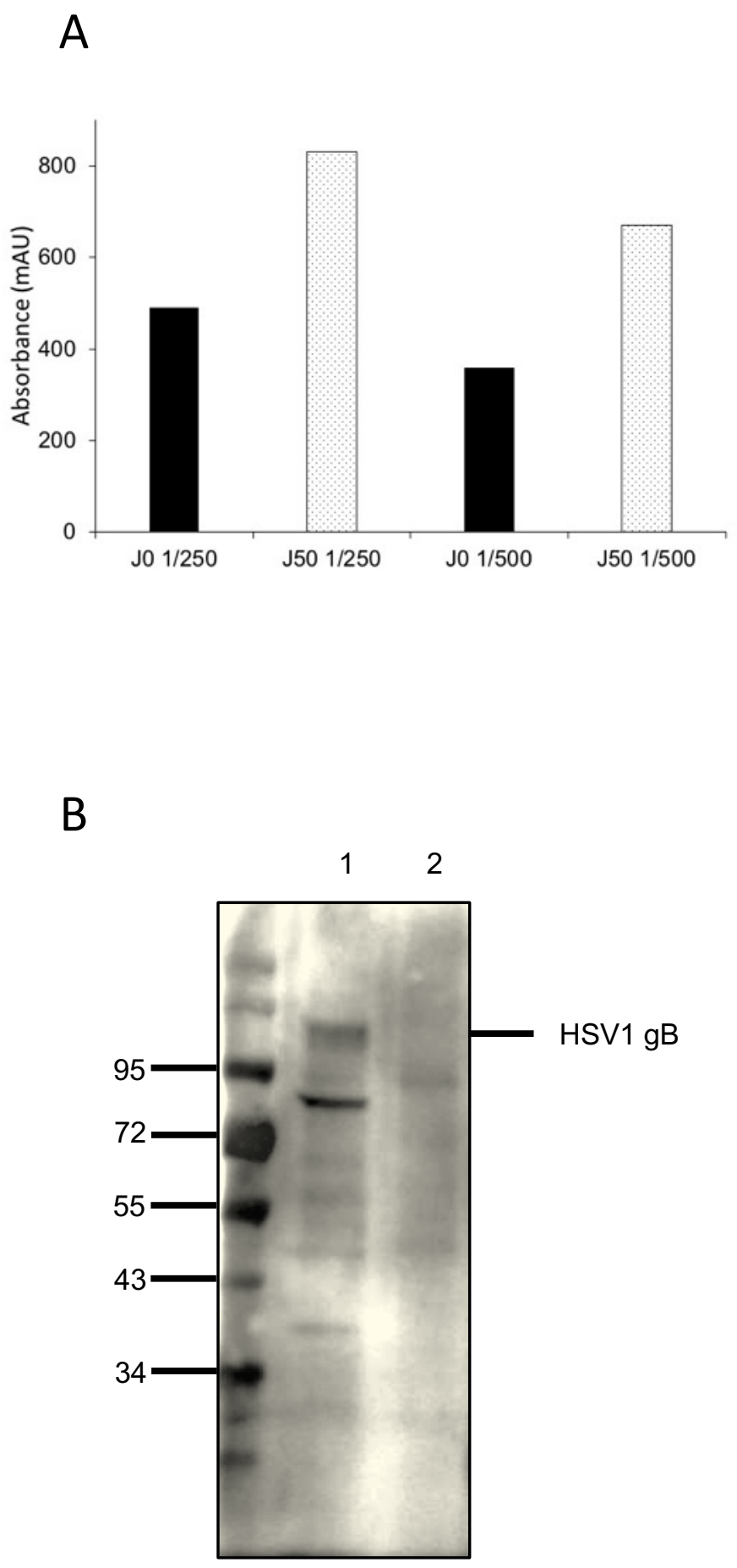
Figure 9

Biophysical studies

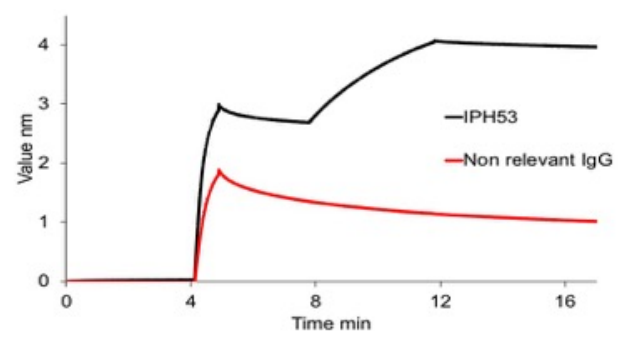

Functional studies

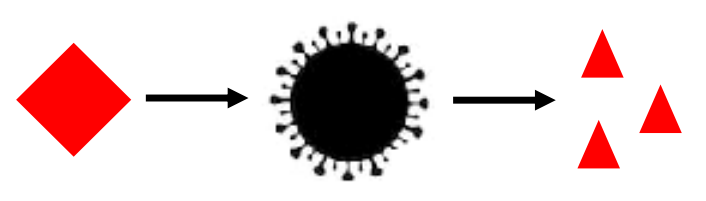

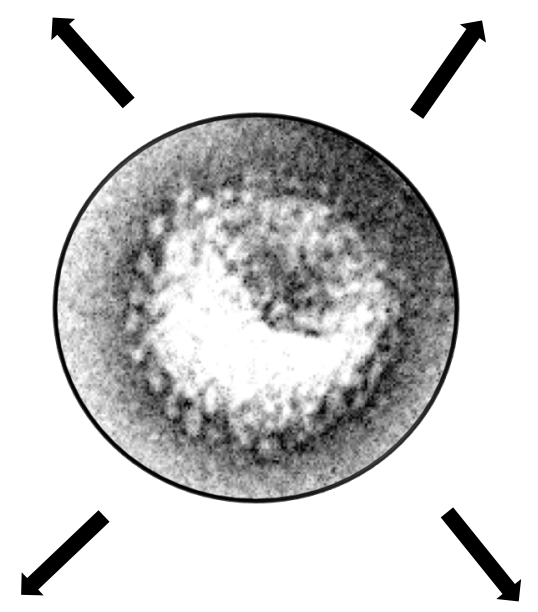

Structure resolution

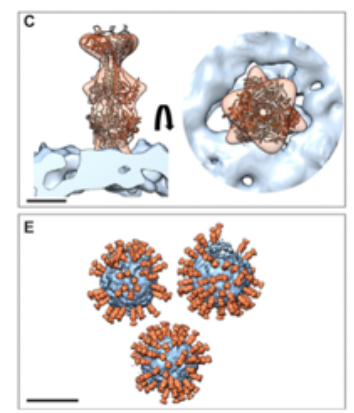

Immunization

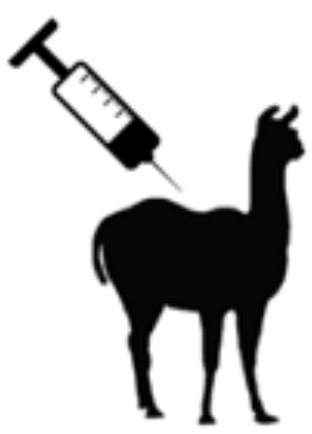

From Zeev-Ben-Mordehai et al Structure 2014 